\title{
Supramolecular Assemblies Obtained by Mixing Different Cyclodextrins and AOT or BHDC Reverse Micelles
}

\author{
O. Fernando Silva, ${ }^{* \dagger}$ N. Mariano Correa, ${ }^{*} \stackrel{\ddagger}{\dagger}$ Juana J. Silber ${ }^{\ddagger}$ Rita H. de Rossi, ${ }^{\dagger}$ \\ and Mariana A. Fernández ${ }^{\dagger}$
}

\begin{abstract}
${ }^{\dagger}$ Instituto de Investigaciones en Físico-Química de Córdoba, INFIQC-CONICET, Facultad de Ciencias Químicas, Departamento de Química Orgánica, Universidad Nacional de Córdoba, Ciudad Universitaria, X5000HUA Córdoba, Argentina

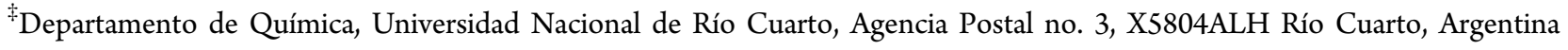

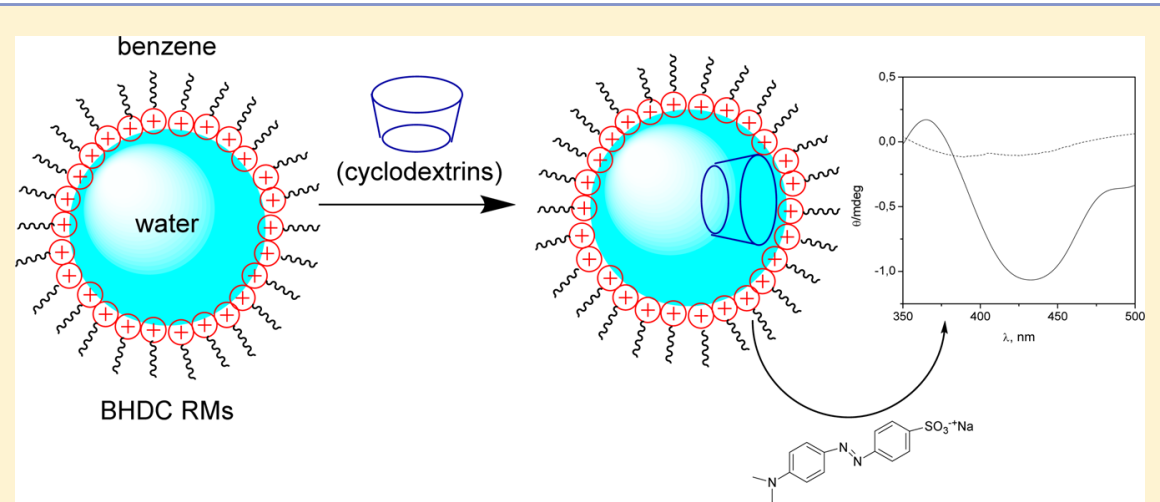

ABSTRACT: In this contribution we show the effect of the surfactant polar head and the external solvent on the incorporation of different cyclodextrins (CDs) $\{\alpha-\mathrm{CD}, \beta-\mathrm{CD}, \gamma-\mathrm{CD}$, decenylsuccinyl- $\beta$-CD (Mod- $\beta$-CD), and hydroxypropyl- $\beta$-CD (hp- $\beta$ $\mathrm{CD})\}$ in different reverse micelles (RMs) \{benzene/sodium 1,4-bis(2-ethylhexyl) sulfosuccinate(AOT)/water, and benzene/ benzyl- $n$-hexadecyldimethylammonium chloride (BHDC)/water $\}$ and compare them with previous results obtained in $n$ heptane/AOT/water RMs. To investigate the different systems, we have used UV-vis spectrophotometry, induced circular dichroism spectroscopy (ICD), and the achiral molecular probe methyl orange (MO). The results show dramatic differences changing the external solvent and the surfactant, which are explained by considering the differences in the RMs interface composition, the water-surfactant interaction, and the CDs' location in the different media investigated. None of the CDs were incorporated into the benzene/AOT/water RMs at any $\left[\mathrm{H}_{2} \mathrm{O}\right] /[$ surfactant $]$ ratio studied $\left(W_{0}\right)$ whereas it was previously shown that Mod- $\beta$-CD and hp- $\beta$-CD could be included in $n$-heptane/AOT/water RMs. However, all of the CDs are incorporated in benzene/BHDC/water RMs at $W_{0}>10$ and hp- $\beta$-CD is dissolved even at $W_{0}=0$. Different from what was found in $n$-heptane/ AOT RMs, in BHDC RMs MO showed ICD signals with two different CDs: Mod- $\beta$-CD and hp- $\beta$-CD. The results are explained by considering the known difference in the interfacial water structure for AOT and BHDC RMs and the electron-rich region on the secondary hydroxyl (wider side of the CDs), which helps to solubilize all CDs in BHDC. This study shows that chiral cyclodextrin could be available for a guest in an organic medium such as the RMs. Therefore we have created a potentially powerful nanoreactor with two different confined regions in the same aggregate: the polar core of the RMs and the chiral hydrophobic cavity of cyclodextrin.

\section{INTRODUCTION}

Several surfactants are able to aggregate in nonaqueous solvents to yield reverse micellar systems (RMs) that are of considerable practical importance in different areas such as detergency, foodstuffs, and cosmetics. ${ }^{1,2}$ Those systems are supramolecular assemblies formed with their polar or charged groups located in the interior (core) whereas their hydrocarbon tails extend into the organic nonpolar solvent medium. ${ }^{1,3,4}$ Interestingly, small solute particles can be dissolved in mainly three different compartments: (a) the external organic solvent, (b) the micellar interface formed by a surfactant monolayer, and (c) the internal water pool. Subsequently, these systems contain aqueous microdroplets entrapped in a film of surfactant and dispersed in a low-polarity bulk solvent. ${ }^{1,5-9}$

Different anionic, cationic, and nonionic surfactants have been employed to prepare RMs. ${ }^{1-4,10,11}$ Among the anionic surfactants that form RMs, the best known are the systems derived from the AOT (sodium 1,4-bis-2-ethylhexylsulfosuccinate, Scheme 1) in different solvents.

It is known that AOT forms spherical RMs in aromatic and hydrocarbons solvents without the addition of a cosurfactant,

Received: November 27, 2013

Revised: February 28, 2014

Published: March 5, 2014 
Scheme 1. Structures of the Surfactants Used in This Work
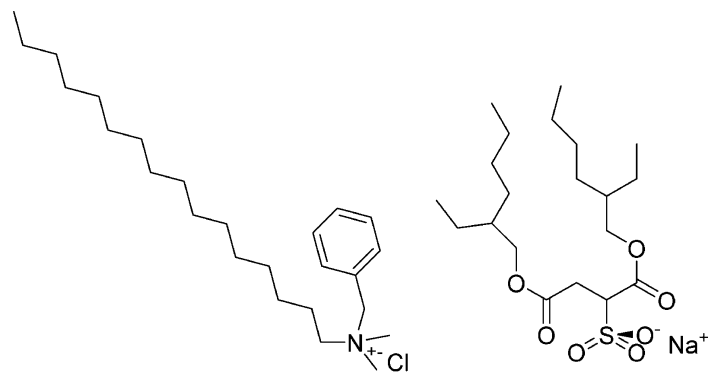

BHDC

AOT

and water can be solubilized up to $W_{0}=\left[\mathrm{H}_{2} \mathrm{O}\right] /[$ surfactant $] \approx$ 60 depending on the external solvent and temperature. Besides, the properties of water change dramatically upon confinement and depend on the $W_{0}$ value. $^{3,4,6,10}$

Cationic surfactant benzyl- $n$-hexadecyldimethylammonium chloride, BHDC (Scheme 1), also forms spherical RMs in benzene without the addition of a cosurfactant, and water can be solubilized up to $W_{0} \approx 25$. $^{12-14}$

With regard to the interfacial properties, the BHDC RMs behave similarly to other RMs systems; namely, the bulk water characteristics are reached only when the amount of water is higher than that necessary for the solvation of the surfactant polar head and the counterion. ${ }^{12,14}$

However, it can be thought that the water properties change in comparison with bulk water simply as a result of the effect of the confinement because it is sequestered on the nanoscale. Previous studies performed by some of us ${ }^{11,15}$ have shown very peculiar and interesting water properties inside RMs that exist not only as a result of the confinement effect but also because of the interaction with the surfactant at the interface. In this way, the water properties are different if water molecules are sequestered in anionic or cationic RM system. This comes out because the water entrapped in the AOT RM systems shows its electron donor ability enhanced in comparison to its bulk water structure. However, the water entrapped in the BHDC RM medium appears to be non-electron-donating as a result of its interaction with the cationic surfactant polar headgroup. In particular, water at the cationic RMs interfaces seems to have its hydrogen bond donor capability enhanced.

Cyclodextrins (CDs) are a group of well-known cyclic oligosaccharides that are capable of forming reversible noncovalent complexes with a wide variety of guests. ${ }^{16}$ These macrocycles consist of several $\alpha$-D-glucopyranose residues (six, seven, or eight rings, named $\alpha$-, $\beta$-, and $\gamma-\mathrm{CD}$, respectively) linked by $\alpha-1,4$ glycosidic bonds (Scheme 2 ).

They have a doughnut-shaped structure in which the cavity has hydrophobic character but the rims, which are surrounded by primary and secondary $\mathrm{OH}$ groups, are hydrophilic.

It is well documented that cyclodextrins form inclusion complexes with most surfactant with high binding constants, ${ }^{17,18}$ and it was demonstrated that they can play important roles in surfactant or surfactant-based assembly systems with many applications such as viscoelasticity control, ${ }^{19}$ DNA decompaction, ${ }^{20,21}$ and protein reconstruction. ${ }^{22,23}$ The roles played by $\mathrm{CDs}$ are, however, complicated and different (or even contradictory) from case to case. For example, it was generally accepted that CDs can destroy micelles in water ${ }^{24,25}$ whereas it was recently revealed that CDs are able to transform mixed surfactant micelles into vesicles. ${ }^{26}$

Scheme 2. Structure of the Different Cyclodextrins Used in This Work
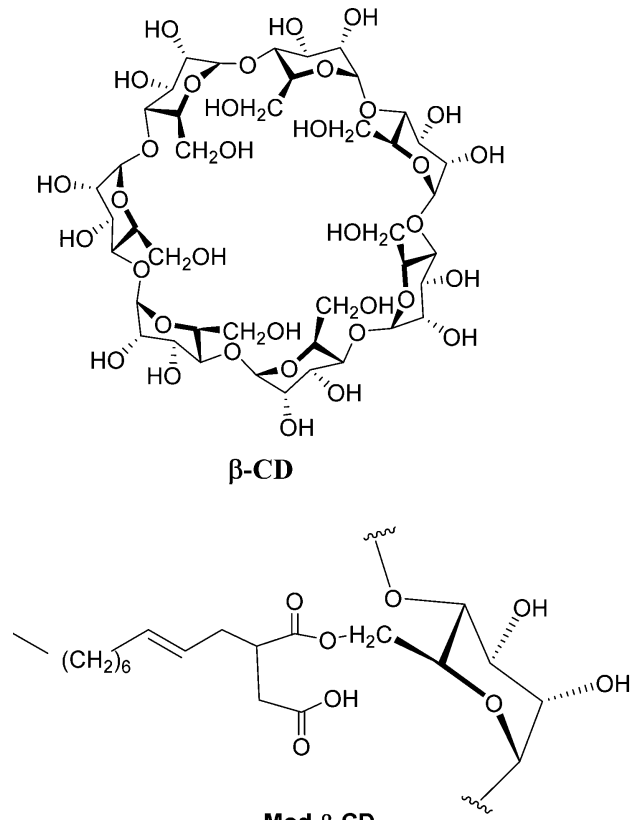

Mod- $\beta-C D$

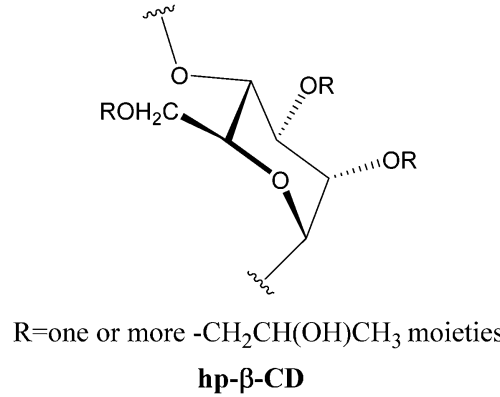

Although the $\mathrm{OH}$ groups on the exterior, in most cases, form $\mathrm{H}$ bonds with water to dissolve the $\mathrm{CDs}$ or $\mathrm{CD}$ complexes (solvation), they can, in some situations, form $\mathrm{CD}-\mathrm{CD} \mathrm{H}$ bonds to induce aggregation and even precipitation (selfassembly). ${ }^{27,28}$ For example, relatively strong $\mathrm{CD}-\mathrm{CD} \mathrm{H}$ bonding in the crystal state was identified and was thought to be responsible for the limited aqueous solubility of natural CDs (in particular, $\beta$-CD) in comparison to that of the comparable acyclic oligosaccharides. Substitution of any of the H-bondforming $\mathrm{OH}$ groups, even by relatively hydrophobic methoxy functions, results in great improvements in aqueous solubility.

With the aim of optimizing the properties of cyclodextrins for a particular use, many substituted CDs have been prepared, and new derivatives continue to be reported. ${ }^{29}$ It has been shown that macrocyclic amphiphiles of hydrophobically modified CDs can form a variety of supramolecular assemblies, including monolayers, multilayers, and Langmuir-Blodgett films at the air-water interface. ${ }^{30}$ Moreover, amphiphilic CDs can be admixed to phospholipids as liposomes, and they can be dispersed into nanospheres, thus exhibiting promising properties for drug encapsulation. ${ }^{31,32}$

Despite their simplicity, RMs provide an excellent method for studying fundamental effects of confinement because unique properties can be created simply by changing the surfactant or the $W_{0}$ value. It is known that a special microenvironment for carrying out a variety of chemical and biochemical reactions is 
found in the polar cores of RMs. ${ }^{33}$ This fact as well as the ability of cyclodextrins to form inclusion complexes with different guest molecules and catalyze chemical reactions as seen in enzymes ${ }^{34,35}$ attracted the researchers to investigate reactions under biomimetic conditions. ${ }^{36-38}$

In trying to link the peculiar catalytic properties of RM and the chiral properties of CDs, we showed in a previous report ${ }^{39}$ that two different cyclodextrins (hydroxypropyl- $\beta$-CD (hp- $\beta$ $\mathrm{CD}$ ) and decenylsuccinyl- $\beta$-CD (Mod- $\beta$-CD)) (Scheme 2) can be incorporated in different zones of RMs made with $n$ heptane/AOT/water. Moreover, using methyl orange (MO) as a chromophoric achiral guest confirmed that the chiral cavity of the CDs is available for the guest inside the RMs. It has been amply demonstrated that the inclusion of achiral guest in a chiral host such as cyclodextrins produces induced circular dichroism (ICD) signals at the wavelengths absorbed by the guest chromophore. ${ }^{40,41}$ Using this approach, we showed that MO produces an ICD signal when it is dissolved in the AOT $\mathrm{RMs}$ only with hp- $\beta$-CD because it resides in the RM water pool as a result of its water solubility. Mod- $\beta$-CD resides on the oil side of the RM interface, probably with AOT tails and/or $n$ heptane molecules inside its cavity, and no ICD signal was detected.

Following along with the idea of characterizing different RMs with CDs that may be used as a chiral nanoreactor, in this article we report the effect of the surfactant polar head and the external solvent on the incorporation of various CDs $(\alpha-\mathrm{CD}, \beta$ $\mathrm{CD}, \gamma$-CD, Mod- $\beta$-CD, and hp- $\beta$-CD) in different RMs (benzene/AOT/water and benzene/BHDC/water). To assess the location of the CDs and the availability of their chiral cavity in the different RMs, we use MO as a molecular probe. The results show that there are dramatic differences in changing the external solvent and the surfactant that were explained by considering the differences in the RMs' interfacial composition, the water-surfactant interaction, and the CDs' location in the different media investigated.

\section{EXPERIMENTAL SECTION}

Materials. Sodium 1,4-bis(2-ethylhexyl) sulfosuccinate (AOT) (Sigma, >99\% purity) was used as received and was kept under vacuum over $\mathrm{P}_{2} \mathrm{O}_{5}$ to minimize $\mathrm{H}_{2} \mathrm{O}$ absorption. The absence of acidic impurities was confirmed through the 1-methyl-8-oxyquinolinium betaine $(\mathrm{QB})$ absorption bands. ${ }^{42}$

Molecular probe methyl orange (MO, Merck), $n$-heptane, and benzene (Merck, HPLC quality) were used as received. $\alpha-\mathrm{CD}, \beta-\mathrm{CD}$, $\gamma$-CD, and hp- $\beta-\mathrm{CD}_{\mathrm{NS}}$ (NS: average degree of substitution $=4.2,5.7$, and 6.9 hydroxypropyl group per cyclodextrin molecule) were purchased from Aldrich or obtained as a gift from Roquette and used as received, but the purity was periodically checked by UV spectroscopy. Mod- $\beta$-CD (average degree of substitution $=1$ carboxylic acid per cyclodextrin molecule) was synthesized in the laboratory by the reaction of 2-decen-1-yl-succinic anhydride and $\beta$ $\mathrm{CD}$ following a procedure reported previously. ${ }^{43}$ Ultrapure water was obtained from a Millipore apparatus.

Methods. The stock solutions of AOT and BHDC in benzene solvent were prepared by mass and volumetric dilution. To obtain optically clear solutions, they were shaken in a sonicating bath. To introduce $\mathrm{MO}$, concentrated solutions of the molecular probe were prepared in acetonitrile (Sintorgan, HPLC quality). An appropriate amount of this solution for obtaining the desired final concentration of molecular probe in the micellar system, $\sim 10^{-5} \mathrm{M}$, was transferred to a volumetric flask, and the acetonitrile was removed by bubbling dry $\mathrm{N}_{2}$. Benzene was added to the residue, and the resulting solution was used to prepare the surfactant-containing samples. The stock surfactant solution at the desired concentration of surfactant was transferred to a flask where the cyclodextrin was previously dissolved in the appropriate amount of water. The water present in the system is expressed as the molar ratio between the polar solvent and the surfactant, $W_{0}=\left[\mathrm{H}_{2} \mathrm{O}\right] /[$ surfactant $]$, and the addition was done using a calibrated microsyringe. The lowest value for $W_{0}\left(W_{0}=0\right)$ corresponds to a system without the addition of water.

General. All of the DLS experiments were carried out at a fixed BHDC concentration of $0.1 \mathrm{M}$. In spite of the fact that the solutions are not at infinite dilution, nevertheless, we consider appropriate to introduce an apparent hydrodynamic diameter $\left(d_{\mathrm{app}}\right)$ in order to make the comparison of our systems as it was used previously. ${ }^{13,44}$ The apparent hydrodynamic diameters of the different BHDC RMs were determined by dynamic light scattering (DLS, Malvern 4700 with a goniometer) with an argon ion laser operating at $488 \mathrm{~nm}$. The cleanliness of the cuvettes used for measurements was crucial to obtaining reliable and reproducible data. ${ }^{45}$ Cuvettes were washed with ethanol and then with doubly distilled water and dried with acetone. Prior to use, the samples were filtered three times to avoid dust or particles present in the original solution using an Acrodisc with a 0.2 $\mu \mathrm{m}$ PTFE membrane (Sigma) for the RM samples. Previous to data acquisition, the samples were equilibrated in the DLS instrument for $10 \mathrm{~min}$ at $32{ }^{\circ} \mathrm{C}$. To obtain valid results from DLS measurements, the knowledge of the system's refractive index and viscosity in addition to well-defined conditions is required. Because BHDC RMs are dilute solutions, we assume that their refractive indices and viscosities are similar to those of neat benzene. Multiple samples at each size were made, and 30 independent size measurements were made for each of them at a scattering angle of $90^{\circ}$ and the polydispersity index of the experiments was always below 0.2. The instrument was calibrated before and during the course of experiments using several different size standards. Thus, the magnitudes obtained by DLS measurements can be taken as statistically meaningful for all of the systems investigated. The algorithm used was CONTIN, and the apparent hydrodynamic diameters reported were weighted by intensity, volume, and/or number because no differences are observed. The DLS experiments show that the polydispersity of the BHDC RMs size is less than $5 \%$. Under our experimental conditions, we have a resolution of $0.5 \mathrm{~nm}$.

The absorption spectra were measured by using a Shimadzu 2101 PC or Shimadzu MultiSpec 1501 spectrometers at $(32.0 \pm 0.1){ }^{\circ} \mathrm{C}$. The wavelength accuracy is $\pm 1 \mathrm{~nm}$, and the photometric accuracy is \pm 0.005 Abs. The induced circular dichroism spectra were measured at the same temperature using Jasco J-810 equipment. The path length used in all spectroscopic experiments was $1 \mathrm{~cm}$.

\section{RESULTS AND DISCUSSION}

Methyl Orange in Benzene/AOT and Benzene/BHDC Reverse Micelles. Because MO was used as a molecular probe, it is worth recalling its previously studied solvatochromism properties. ${ }^{39}$ The Kamlet-Taft's solvatochromic comparison method $(\mathrm{KTSCM})^{46}$ analysis shows that the MO absorption spectrum shifts to longer wavelength with increasing solvent polarity-polarizability $\left(\pi^{*}\right)$ and the hydrogen donor ability $(\alpha)$ of the medium. MO is almost 3 times more sensitive to the $\pi^{*}$ parameter than to the $\alpha$ parameter, which is reasonable considering that the molecule is very polarizable as a result of extended resonance.

We showed in our previous report ${ }^{39}$ that MO in $n$-heptane/ AOT RMs is most likely situated at the RM interface and not at the water pool despite the negative charge of the AOT and the molecular probe. It was suggested that the high ionic strength inside AOT RM favors the MO ion pairing with the sodium counterions (from surfactant and molecular probe) and thus decreases the effective charge of the MO molecules. As a consequence, MO has a greater tendency to reside at the AOT RM interface.

Now we are interested in studying the properties of several RMs interfaces and the effect of the external solvent, thus we 
performed different experiments to investigate the location of $\mathrm{MO}$ in AOT and BHDC RMs using benzene as an external nonpolar medium. The surfactant concentration was varied at constant $W_{0}(0$ and 10$)$ in the RM systems without the addition of cyclodextrins. The concentration range used was 0.001-0.3 M for AOT and 0.005-0.2 M for BHDC. The MO absorption spectrum (results not shown) did not change with the surfactant concentration. Similar results were observed previously in $n$-heptane AOT RMs ${ }^{39}$ and suggest that because $\mathrm{MO}$ is not soluble in the organic pseudophase it resides exclusively inside RMs without partitioning to the organic solvent. 4,10

The situation was quite different when the amount of water was varied at constant surfactant concentration. Figure $1 \mathrm{~A}, \mathrm{~B}$ shows the $\mathrm{MO}$ absorption spectra at $[\mathrm{BHDC}]=0.1 \mathrm{M}$ and [AOT $]=0.3 \mathrm{M}$ as a function of $W_{0}$.

It can be seen that upon water addition the absorption maxima slightly change (from 417 to $419 \mathrm{~nm}$ ) in benzene/ BHDC RMs but never reach the value of the maximum in pure water $(467 \mathrm{~nm})$, and the same behavior was observed in
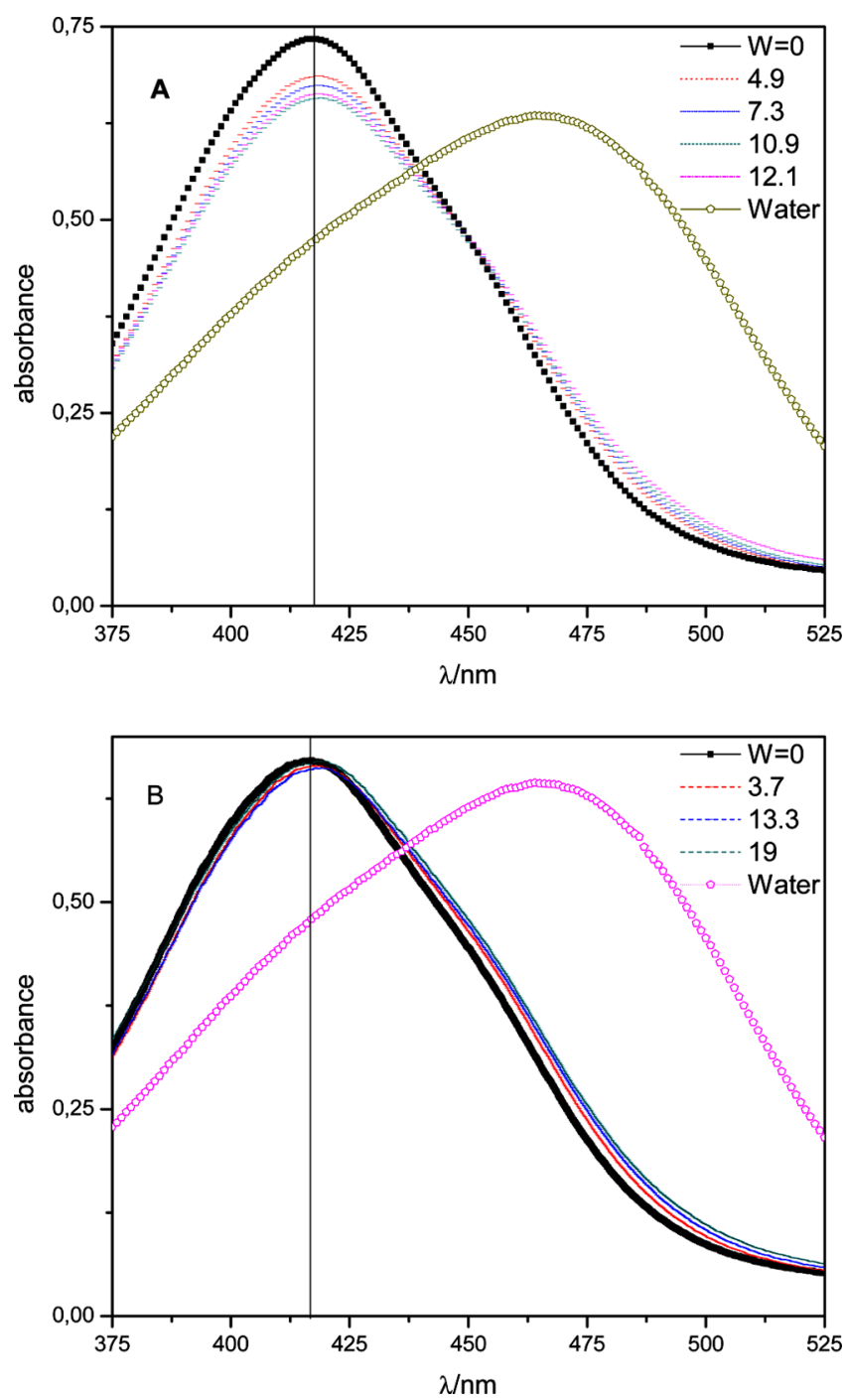

Figure 1. (A) Absorption spectra of MO in benzene/AOT (0.3 M) RMs at different $W$ values. (B) Absorption spectra of MO in benzene/ $\operatorname{BHDC}(0.1 \mathrm{M}) \mathrm{RMs}$ at different $W$ values, $[\mathrm{MO}]=2 \times 10^{-5} \mathrm{M}$. Temperature $32{ }^{\circ} \mathrm{C}$. The line at $417 \mathrm{~nm}$ is only a guide for the eye.
benzene/AOT RMs. A more notable red shift was previously observed for $n$-heptane/AOT RMs, ${ }^{39}$ and it was attributed to strong hydrogen bond complexes between water and the azo nitrogen group of $\mathrm{MO}$, which causes a new band to develop at around $\lambda=467 \mathrm{~nm} .{ }^{39}$ Moreover, major changes in the absorption maxima (from 402 to $408 \mathrm{~nm}$ ) occur up to $W=10$ and after that remain constant and never reach the value of the maximum in pure water. Hence, MO at the $n$-heptane/AOT RMs interacts through hydrogen bonds with the water of hydration up to $W=10$ and then remains anchored at the interface because of this interaction. The situation is different when benzene is used as the external solvent (Figure 1). Herein, our results indicate that MO barely interacts with the water of hydration even up to the highest $W_{0}$ values that the systems can accept without collapsing, namely, $W_{0} \approx 12$ for AOT and $W_{0} \approx 19$ for BHDC RMs. These results were expected, considering what was previously found for $\mathrm{AOT}^{42}$ and $\mathrm{BHDC}^{13} \mathrm{RMs}$ formed in benzene, where the watersurfactant interaction is weak and water penetrates toward the oil side of the interface. Consequently, $\mathrm{MO}$ in the anionic benzene/AOT RMs still interacts with the interfacial water but less than when $n$-heptane is used as the external solvent. In the cationic BHDC RMs, because MO bears a negative charge in its moiety and $\mathrm{BHDC}$ has a positive charge in its polar headgroup, it is very likely that the coulomb interaction anchors $\mathrm{MO}$ in the interface and is not disrupted with the increase of water in the $\mathrm{RM}$ with the consequent independence of the spectra with the $W_{0}$ value. Moreover, considering the maxima absorption wavelength of $\mathrm{MO}$ in benzene/AOT RMs or benzene/ BHDC RMs (both are at $417 \mathrm{~nm}$ ), we concluded that MO is located in the oil side of the RM interface sensing a less polar medium than in $n$-heptane/AOT. ${ }^{39}$ In this last system, the absorption band at $467 \mathrm{~nm}$ showed that $\mathrm{MO}$ is in the water side of the micelle interface, always sensing a more polar and more hydrogen bond donor environment, as was invoked for other molecular probes. ${ }^{4,10}$ To provide evidence about the interactions at the interface, we have plotted the ratio of Abs ${ }^{\lambda 417 \mathrm{~nm}} /$ $\mathrm{Abs}^{\lambda 467 \mathrm{~nm}}$ as a function of $W_{0}$ (Figure 2), keeping in mind that the absorbance at $\lambda=467 \mathrm{~nm}$ corresponds to $\mathrm{MO}$ interacting through hydrogen bonds with water. ${ }^{39}$

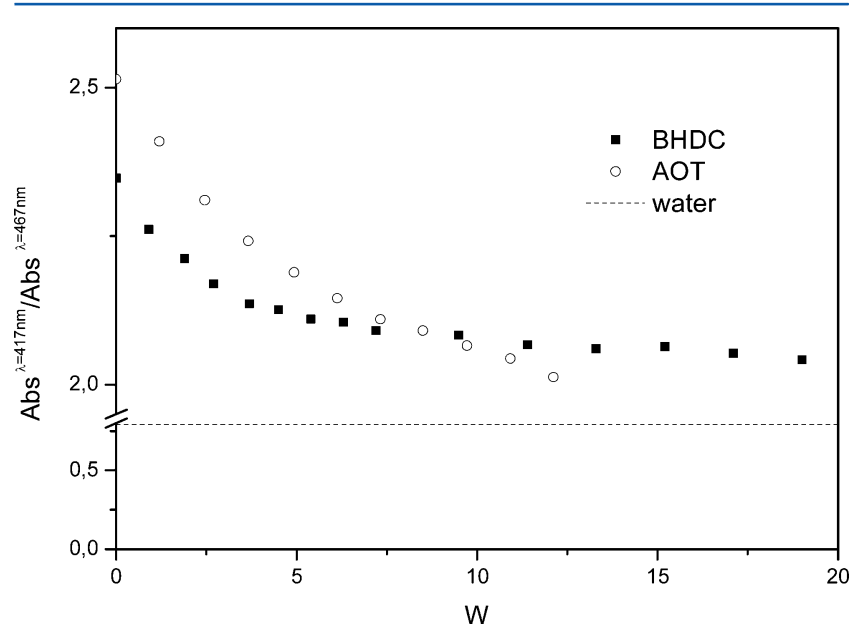

Figure 2. Variation of $\mathrm{Abs}^{\lambda=417 \mathrm{~nm}} / \mathrm{Abs}^{\lambda=467}$ as a function of $W_{0}$ in benzene/BHDC/water (squares), benzene/AOT/water (open circles), water (dashed line), $[\mathrm{AOT}]=0.3 \mathrm{M},[\mathrm{BHDC}]=0.1 \mathrm{M}$, and $[\mathrm{MO}]=2 \times 10^{-5} \mathrm{M}$. 
As can be seen, MO in AOT or BHDC RMs practically does not interact with the interfacial water through hydrogen bonds because it never reaches the value that it has in pure water.

The results reported here and previously ${ }^{39}$ indicate that $\mathrm{MO}$ in AOT RMs is mostly associated with the $\mathrm{Na}^{+}$counterion, whereas in BHDC RMs MO interacts with the positive charge of the surfactant.

Cyclodextrins in Reverse Micelles. After the evaluation of the MO location in the anionic and cationic RMs, the next step was to investigate if CDs can be solubilized in the different RM media.

In benzene/AOT RMs, it turns out that native $(\alpha-, \beta$-, and $\gamma$ $\mathrm{CD}$ ) and modified (Mod- $\beta$-CD and hp- $\beta$-CD) cyclodextrins (Scheme 2) could not be solubilized in the RM system at any $W_{0}$ values investigated. It seems that the hydrogen bond interaction between the CD hydroxyl group and the AOT polar headgroup, although a very effective hydrogen bond acceptor, ${ }^{4,42}$ is not strong enough to solubilize these CDs when benzene is used as a nonpolar medium. Also, it is possible that some benzene molecules are dissolved in the water pool of the AOT RMs where hp- $\beta$-CD should be located, with a consequent decrease in the hp- $\beta$-CD water solubility, thus preventing the encapsulation of these CDs. These results are very different from those obtained in $n$-heptane/AOT RMs where it was possible to solubilize hp- $\beta-\mathrm{CD}_{5.7}$ (at $W_{0}>10$ and $[\mathrm{hp}-\beta-\mathrm{CD}]<4.75 \mathrm{mM})$ and Mod- $\beta-\mathrm{CD}$ at $W_{0}>20([$ Mod- $\beta$ $\mathrm{CD}<1 \mathrm{mM}) .^{39}$ Moreover, when $n$-heptane is the external nonpolar solvent, water molecules interact through hydrogen bonds with the AOT sulfonate group without penetration to the interface and making a more polar, stronger hydrogen bond donor environment where both CDs were solubilized in different regions within the aggregate. ${ }^{4}$

Surprisingly, in BHDC RMs all of the different CDs used were solubilized at $W_{0}=10, \mathrm{BHDC}=0.1 \mathrm{M}(\alpha, \beta, \gamma-\mathrm{CD}$ and Mod- $\beta$-CD at concentrations below $1 \mathrm{mM}$ and hp- $\beta$-CD at concentrations below $4 \mathrm{mM})$. In addition, hp- $\beta-\mathrm{CD}_{5.7}$ and hp$\beta-\mathrm{CD}_{6.3}$ were solubilized even at $W_{0}=0$ (at concentrations below $4 \mathrm{mM}$ ). To explain these results, we consider the following features: (i) Studies of electrostatic surface potential (ESP) suggest that the rim of the CD's wider opening exhibits relatively hydrophilic character with low potential values and an electron-rich region on the secondary hydroxyl (wider side) ${ }^{47}$ (ii) the different interfacial water structure found in BHDC RMs in comparison to that found in AOT RMs explained above. ${ }^{11,15}$ As water molecules interact with BHDC through its nonbonding electrons, its hydrogen bond donor capability is enhanced, which may favor the interaction with different CDs in the same way that helps to anchor enzymes at the BHDC RM interface. ${ }^{11,48}$ In addition, the high electronic density at the secondary rim of CDs mentioned above may favor the electrostatic interaction between the positive polar headgroup of $\mathrm{BHDC}$, thus helping to solubilize CDs in these RMs.

The fact that hp- $\beta-\mathrm{CD}_{5.7}$ and $\mathrm{hp}-\beta-\mathrm{CD}_{6.3}$ can be incorporated into RMs even in the absence of added water $(W=0)$ may be attributed to the interaction of the $\mathrm{OH}$ groups in the lateral chain with the polar headgroup of the surfactant. The flexibility of the lateral chain allows the proper orientation of the electron donor $\mathrm{OH}$ groups that result in stabilizing interactions. It appears that a minimum number of hydroxypropyl groups are required, which is quite reasonable considering the low free energy gained for each electron donor-acceptor bond formed.
Herein, the question of the location of CDs inside BHDC RMs remains because at $W_{0}>10$ they can be solubilized at the $\mathrm{RM}$ interface or in the water pool. We used two different approaches: dynamic light scattering (DLS) and ICD experiments using $\mathrm{MO}$ as a molecular probe. These experiments are discussed in the following sections.

Dynamic Light Scattering Experiments. Because the addition of $\mathrm{CD}$ to the RMs could change the characteristics of the solution, ${ }^{49}$ we performed DLS experiments with RMs containing only water and others loaded with the CD solutions. It is known that the increase in the RM droplet size is associated with molecules that reside at the RM interface, changing the effective packing parameter of the surfactants, $v /$ $a l_{c}$ in which $v$ and $l_{c}$ are the volume and length of the hydrocarbon chain, respectively, and $a$ is the surfactant headgroup area. The RMs sizes are larger when the surfactant packing parameter values are smaller. ${ }^{4,50}$ Moreover, we recently showed that molecules that do not interact with the interface upon encapsulation do not change the RM droplet size values. ${ }^{51}$

DLS experiments afforded interesting results. At $W_{0}=10$, $\mathrm{BHDC}=0.1 \mathrm{M}$, and $\alpha, \beta, \gamma-\mathrm{CD}$ and Mod- $\beta$-CD showed that there is an increase in the apparent hydrodynamic diameter $\left(d_{\text {app }}\right.$ ) from $9.7 \mathrm{~nm}$ (without CDs) to $11.7 \mathrm{~nm}$ (at a $1 \mathrm{mM}$ concentration of $\mathrm{CD}$ ) with the same polydispersity. This result suggests that the CDs are anchored to the interface, interacting with water and thus increasing the surfactant headgroup, which results in a decrease in the packing parameter.

On the contrary, hp- $\beta-\mathrm{CD}_{5.7}$ (at $4 \mathrm{mM}$ concentration) encapsulated inside the BHDC RMs shows a droplet size value that is significantly lower $(7.2 \mathrm{~nm})$ than RMs without cyclodextrins. This behavior can be explained by considering that the favorable hydrogen bond interaction between the $\mathrm{OH}$ groups of the lateral chain in hp- $\beta$-CD can remove water from the interface, thus decreasing the $a$ value and consequently the droplet size. It has been suggested that this dehydration behavior plays a large role in the interaction between hp- $\beta$-CD and tauroconjugated and glycoconjugated bile salts. ${ }^{52}$

At $W=0$, the BHDC RMs are small $(2 \pm 1 \mathrm{~nm})$ and the size is very polydisperse, precluding any reasonable conclusions.

In conclusion, all of the CDs seem to be located on the water side of the BHDC RM interface, and none of them are located in the water pool, was the case for hp- $\beta$-CD in $n$-heptane/AOT RMs. ${ }^{39}$ Scheme 3 summarizes the above discussion.

MO in BHDC RMs: Induced Circular Dichroism (ICD) Spectra Studies. To obtain a clear idea of the location of the CDs inside BHDC RMs, we have performed ICD experiments using $\mathrm{MO}$ as a molecular probe. We expected that the combination of these experimental results with those obtained through DLS measurements could provide information about the position of the chiral cyclodextrin in the microheterogeneous system.

It has been amply demonstrated that the association of a chromophoric achiral guest with a chiral host such as cyclodextrin gave ICD signals at the wavelengths absorbed by the guest chromophore. ${ }^{40,53-55}$ The sign and magnitude of the ICD signals are critical functions of the relative orientation of the chromophore in the host cavity. Empirical rules to relate the ICD signals to the structure of the complexes of CD with a guest were proposed by Harata ${ }^{40}$ and Kodaka. ${ }^{56}$ These widely accepted rules predict that when a guest molecule is accommodated inside the cavity with its transition dipole moment parallel to the host axis a positive ICD signal is 
Scheme 3. Cartoon Representation of the Estimated Different Locations for CD in BHDC RMs ${ }^{a}$

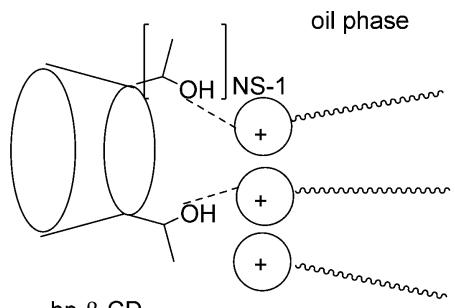

$\mathrm{hp}-\beta-C D_{\mathrm{NS}}$

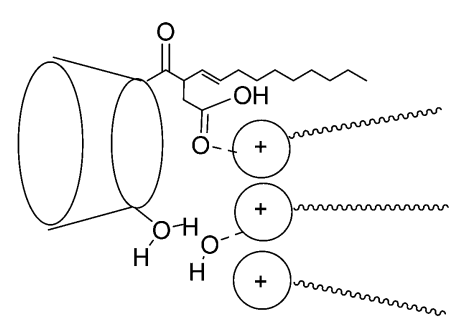

Mod- $\beta-C D$

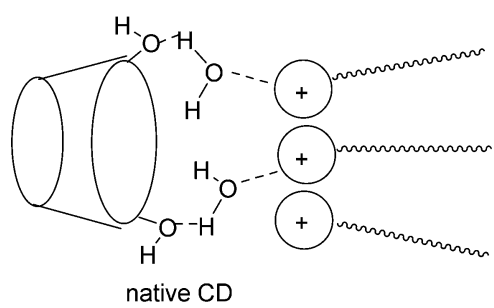

${ }^{a}$ For simplicity, the MO bound to the interface is not represented.

observed, whereas when the transition moment is perpendicular to the axis, a negative ICD signal appears (Harata's rule). ${ }^{40}$ In contrast, for a guest located outside the cavity, completely opposite ICD behavior is expected (i.e., the transition dipole moment parallel to the host axis affords a negative ICD signal whereas the perpendicular transition gives the opposite signal (Kodaka's rule $)^{56-58}$ ).

In our previous work with $n$-heptane/AOT RMs, ${ }^{39}$ we showed that hp- $\beta$-CD can induce an appreciable positive CD signal at $\lambda=465 \mathrm{~nm}$, which corresponds to the $\pi-\pi^{*}$ transition band of the azo group of MO. The positive ICD signal with hp$\beta$-CD indicates that MO penetrates the cavities in the longitudinal direction, where the $\pi-\pi^{*}$ transition moment of the azochromophore in $\mathrm{MO}$ is parallel to the host axis. ${ }^{56,59,60}$ Also, it was shown that the complex is formed in the water pool of the AOT RMs.

The situation is quite different for the benzene/BHDC RMs. Figure 3 shows a typical ICD spectra of MO in the absence and presence of hp- $\beta-\mathrm{CD}_{5.7}$ or Mod- $\beta-\mathrm{CD}$ at $W_{0}=10$. Also at $W_{0}=$ $0, \mathrm{MO}$ gave an ICD signal with hp- $\beta-\mathrm{CD}_{5.7}$ (results not shown). It must be pointed out that there is no ICD signal in the absence of CDs. Herein it is worthy to mention that in $n$ heptane/AOT RMs Mod- $\beta$-CD does not give an ICD signal, and it was explained by considering that the $\mathrm{CD}$ cavity might be occupied with the surfactant chain. ${ }^{39}$

In BHDC RMs, we suggested above that the CDs interact with $\mathrm{BHDC}$ at the interface through its wider rim and the lateral $\mathrm{OH}$ or carboxylic group in $\mathrm{HP}-\beta-\mathrm{CD}$ or Mod- $\beta-\mathrm{CD}$, respectively, and it is likely that $\mathrm{MO}$ is also at the interface interacting with BHDC and located close to the $\mathrm{CD}$ wall, therefore in a chiral environment.

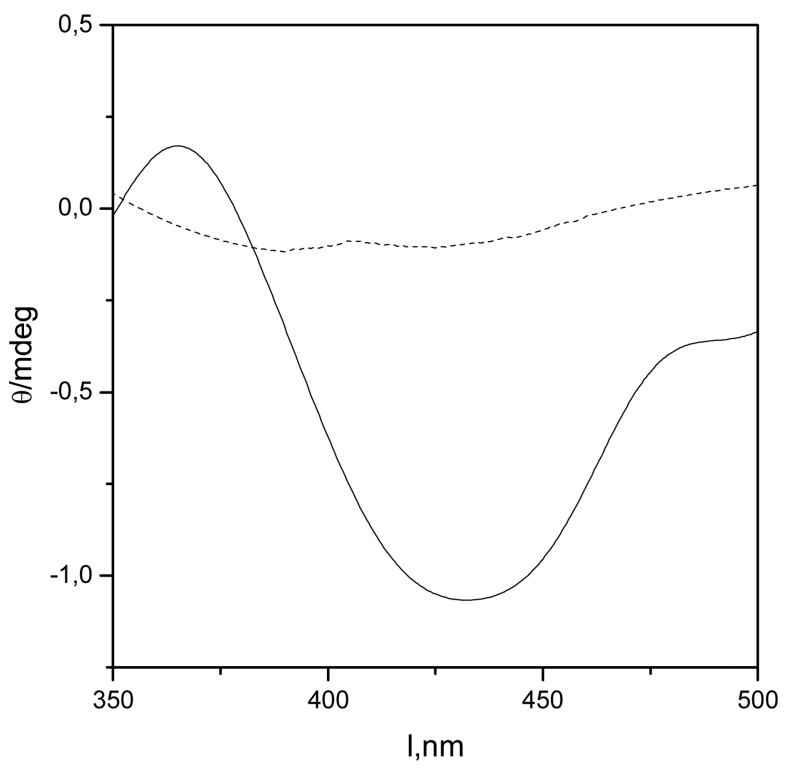

Figure 3. ICD spectrum of $\mathrm{MO}$ in the absence (dashed line) and presence (solid line) of hp- $\beta$-CD in BHDC RMs. [BHDC] $=0.1 \mathrm{M}, W$ $=10,[\mathrm{hp}-\beta-\mathrm{CD}]=4 \times 10^{-3} \mathrm{M}$, and $[\mathrm{MO}]=2 \times 10^{-5} \mathrm{M}$. Temperature: $32{ }^{\circ} \mathrm{C}$.

As can be seen (Figure 3), the ICD signal is negative for $\mathrm{MO}$ with $\mathrm{CDs}$ in BHDC RMs, which suggests that the structure of the complex is appreciably different compared to that observed in AOT RMs.

To confirm that the MO-CD complexation takes place at the $\mathrm{RM}$ interface and not in the water pool, we have performed a UV-visible study. Figure 4 shows the absorption spectra of $\mathrm{MO}$ in BHDC RMs at $\mathrm{BHDC}=0.1 \mathrm{M}$ and $W_{0}=10$ with and without the presence of hp- $\beta-\mathrm{CD}$ and Mod- $\beta-\mathrm{CD}$.

The absorption spectra show that the $\lambda_{\max }$ of the solution containing $\mathrm{CD}$ is the same as that in its absence, which suggests that the complex is formed at the RM interface and not in the water pool, where the $\lambda_{\max }$ of the complex is around $450 \mathrm{~nm} .{ }^{39}$

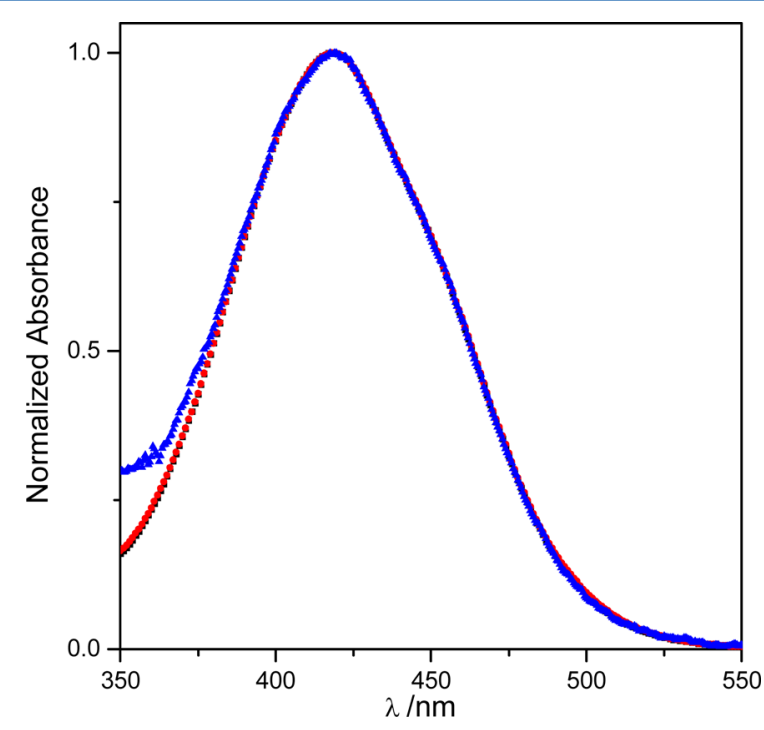

Figure 4. Normalized absorption spectra of $\mathrm{MO}$ in the absence (black) and presence of hp- $\beta$-CD (red) or Mod- $\beta$-CD (blue) in BHDC RMs at $W_{0}=10$. [hp- $\left.\beta-\mathrm{CD}\right]=4 \times 10^{-3} \mathrm{M}$, [Mod- $\left.\beta-\mathrm{CD}\right]=1$ $\times 10^{-3} \mathrm{M}$, and $[\mathrm{BHDC}]=0.1 \mathrm{M}$. 
It is interesting that the spectra are the same at any $\mathrm{CD}$ concentration investigated in the range of 0.0 to $4.0 \times 10^{-3} \mathrm{M}$. This result suggests that MO is not inside the cavity.

However, no ICD signals were detected with any of the native cyclodextrins under any conditions, which probably reflects the fact that the strong interaction of $C D$ with the interface may act as a cup in one of the $\mathrm{CD}$ rims, preventing the access of MO to the cavity, and the packing is not favorable to the interaction with the external walls of CDs.

This result is very challenging because now we have a chiral supramolecular structure (the cyclodextrins) inside an organized medium such as the BHDC RMs, giving a novel medium very different from that shown for AOT RMs to use as a nanoreactor. The complexation process between the guest and the $\mathrm{CDs}$ is at the interface, where the RMs have a greater catalytic effect on chemical reactions. ${ }^{4,11,48}$

\section{CONCLUSIONS}

This study presents the investigation of novel BHDC RMs made with the BHDC surfactant, water, benzene and the incorporation of different CDs: $\alpha$-CD, $\beta$-CD, $\gamma$-CD, Mod- $\beta$-CD (decenylsuccinyl- $\beta-\mathrm{CD}$ ), and hp- $\beta$-CD (hydroxypropyl- $\beta-\mathrm{CD}$ ). The results show that RMs with chiral molecules such as cyclodextrins in the same aggregates can be formed and the CD interacts with an external guest in an organic medium such as the RMs.

Using DLS techniques, we demonstrated that CDs reside at the interface, whereas the native $C D$ s interact strongly with the interface, and for the modified CDs, hp- $\beta$-CD and Mod- $\beta$-CD, the side chains anchor the CDs at the interface as summarized in Scheme 3. These different arrays of CDs at the interface exhibit remarkable behavior in interacting with the external guest. Using absorption UV-visible spectroscopy and induced circular dichroism (ICD) and methyl orange as a molecular probe, we concluded that the array of native CDs probably acting as a cup in one of the $\mathrm{CD}$ rims prevents the access of MO to the cavity. The situation is different for modified CDs where these arrays allow interaction with the $\mathrm{MO}$ and give an opposite ICD signal, as was previously observed in $n$-heptane/ AOT RMs. From the UV studies, we show that $\mathrm{MO}$ is not inside the cavity. On the contrary, as was observed in $n$ heptane/AOT RMs, MO penetrates the cavities in the longitudinal direction.

Also it is demonstrated that the change in the external solvent and the surfactant have dramatic consequences regarding the interaction of CDs with RMs. None of the CDs were incorporated in the benzene/AOT/water RMs at any $W_{0}$ value studied, whereas it was previously shown that Mod- $\beta$ $\mathrm{CD}$ and hp- $\beta$-CD could be included in $n$-heptane/AOT/water RMs. However, all of the CDs are incorporated in benzene/ $\mathrm{BHDC} /$ water RMs at $W_{0}>10$, and hp- $\beta$-CD is dissolved even at $W_{0}=0$.

This study allows an exploration of the functionality of the interfaces, and it may be possible that these systems can be used as nanoreactors in asymmetric synthesis, a topic that we are currently investigating.

\section{AUTHOR INFORMATION}

\section{Corresponding Authors}

*E-mail: fersilva@fcq.unc.edu.ar.

*E-mail: mcorrea@exa.unrc.edu.ar.

\section{Notes}

The authors declare no competing financial interest.

\section{ACKNOWLEDGMENTS}

Financial support from the Consejo Nacional de Investigaciones Cientificas y Técnicas (CONICET), Universidad Nacional de Río Cuarto, Universidad Nacional de Córdoba, Fundación Antorchas, and Agencia Nacional de Promoción Cientifica y Técnica is gratefully acknowledged. O.F.S., J.J.S., R.H.d.R., M.A.F., and N.M.C. hold research positions at CONICET.

\section{REFERENCES}

(1) Moulik, S. P.; Paul, B. K. Structure, dynamics and transport properties of microemulsions. Adv. Colloid Interface Sci. 1998, 78, 99195.

(2) Uskokovic, V.; Drofenik, M. Reverse micelles: inert nano-reactors or physico-chemically active guides of the capped reactions. Adv Colloid Interface Sci. 2007, 133, 23-34.

(3) De, T. K.; Maitra, A. Solution behavior of aerosol OT in nonpolar solventes. Adv. Colloid Interface Sci. 1995, 59, 95-193.

(4) Silber, J. J.; Biasutti, M. A.; Abuin, E.; Lissi, E. Interactions of small molecules with reverse micelles. Adv. Colloid Interface Sci. 1999, $82,189-252$

(5) Fendler, J. H. Membrane Mimetic Chemistry; Wiley: New York, 1982.

(6) Pileni, M. P. Structure and Reactivity of Reverse Micelles; Elsevier: New York, 1989.

(7) Zhang, X.; Chen, Y.; Liu, J.; Zhao, C.; Zhang, H. Investigation on the structure of water/AOT/IPM/alcohols reverse micelles by conductivity, dynamic light scattering, and small angle X-ray scattering. J. Phys. Chem. B 2012, 116, 3723-3724.

(8) Sedgwick, M.; Cole, R. L.; Rithner, C. D.; Crans, D. C.; Levinger, N. E. Correlating proton transfer dynamics to probe location in confined environments. J. Am. Chem. Soc. 2012, 134, 11904-11907.

(9) Levinger, N. E.; Rubenstrunk, L. C.; Baruah, B.; Crans, D. C. Acidification of reverse micellar nanodroplets by atmospheric pressure $\mathrm{CO}_{2}$. J. Am. Chem. Soc. 2011, 133, 7205-7214.

(10) Correa, N. M.; Silber, J. J.; Riter, R. E.; Levinger, N. E. Nonaqueous polar solvents in reverse micelle systems. Chem. Rev. 2012, 112, 4569-4602.

(11) Quintana, S. S.; Moyano, F.; Falcone, R. D.; Silber, J. J.; Correa, N. M. Characterization of multifunctional reverse micelles' interfaces using hemicyanines as molecular probes. II: Effect of the surfactant. J. Phys. Chem. B 2009, 113, 6718-6724.

(12) McNeil, R.; Thomas, J. K. Benzylhexadecyldimethylammonium chloride in microemulsions and micelles. J. Colloid Interface Sci. 1981, $83,57-65$.

(13) Agazzi, F. M.; Falcone, R. D.; Silber, J. J.; Correa, N. M. Solvent blends can control cationic reversed micellar interdroplet interactions. the effect of $n$-heptane:benzene mixture on BHDC reversed micellar interfacial properties: droplet sizes and micropolarity. J. Phys. Chem. B 2011, 115, 12076-12084.

(14) Grand, D.; Dokutchaev, A. Does the interfacial potential control the charge separation efficiency in reverse micellar media? J. Phys. Chem. B 1997, 101, 3181-3186.

(15) Blach, D.; Correa, N. M.; Silber, J. J.; Falcone, R. D. Interfacial water with special electron donor properties: effect of watersurfactant interaction in confined reversed micellar environments and its influence on the coordination chemistry of a copper complex. J. Colloid Interface Sci. 2011, 355, 124-130.

(16) D’Souza, V. T.; Lipkowitz, K. B. Cyclodextrins: introduction. Chem. Rev. 1998, 98, 1741-1742.

(17) Jiang, L.; Yan, Y.; Huang, J. Versatility of Cyclodextrins in SelfAssembly Systems of amphiphiles. Adv. Colloid Interface Sci. 2011, 169, $13-25$. 
(18) Carlstedt, J.; Bilalov, A.; Krivtsova, E.; Olsson, U.; Lindman, B. Cyclodextrin - surfactant coassembly depends on the cyclodextrin ability to crystallize. Langmuir 2012, 28, 2387-2394.

(19) Talwar, S.; Harding, J.; Oleson, K. R.; Khan, S. Surfactantmediated modulation of hydrophobic interactions in associative polymer solutions containing cyclodextrin. Langmuir 2009, 25, 794802.

(20) González-Pérez, A.; Carlstedt, J.; Dias, R. S.; Lindman, B. Cyclodextrins in DNA decompaction. Colloids Surf., B 2010, 76, 2027.

(21) González-Pérez, A.; Dias, R. S.; Nylander, T.; Lindman, B. Cyclodextrin-surfactant complex: a new route in DNA decompaction. Biomacromolecules 2008, 9, 772-775.

(22) Li, L.; Nachtergaele, S.; Seddon, A. M.; Tereshko, V.; Ponomarenko, N.; Ismagilov, R. F. Simple host-guest chemistry to modulate the process of concentration and crystallization of membrane proteins by detergent capture in a microfluidic device. $J$. Am. Chem. Soc. 2008, 130, 14324-14328.

(23) Signorell, G. A.; Kaufmann, T. C.; Kukulski, W.; Engel, A.; Rémigy, H.-W. Controlled 2D crystallization of membrane proteins using methyl- $\beta$-cyclodextrin. J. Struct. Biol. 2007, 157, 321-328.

(24) Fernández, M. A.; de Rossi, R. H. Changing mechanisms in the $\beta$-cyclodextrin-mediated hydrolysis of phenyl esters of perfluoroalkanoic acids. J. Org. Chem. 2003, 68, 6887-6893.

(25) Xing, H.; Lin, S.-S.; Yan, P.; Xiao, J.-X. Demicellization of a mixture of cationic-anionic hydrogenated/fluorinated surfactants through selective inclusion by $\alpha$ - and $\beta$-cyclodextrin. Langmuir 2008, 24, 10654-10664.

(26) Jiang, L.; Yu, C.; Deng, M.; Jin, C.; Wang, Y.; Yan, Y.; Huang, J. Selectivity and stoichiometry boosting of $\beta$-cyclodextrin in cationic/ anionic surfactant systems: when host-guest equilibrium meets biased aggregation equilibrium. J. Phys. Chem. B 2010, 114, 2165-2174.

(27) Messner, M.; Kurkov, S. V; Jansook, P.; Loftsson, T. Selfassembled cyclodextrin aggregates and nanoparticles. Int. J. Pharm. 2010, 387, 199-208.

(28) Puskás, I.; Schrott, M.; Malanga, M.; Szente, L. Characterization and control of the aggregation behavior of cyclodextrins. J. Incl. Phenom. Macrocycl. Chem. 2013, 75, 269-276.

(29) Kauscher, U.; Stuart, M. C. A.; Drücker, P.; Galla, H.-J.; Ravoo, B. J. Incorporation of amphiphilic cyclodextrins into liposomes as artificial receptor units. Langmuir 2013, 29, 7377-7383.

(30) Vico, R. V; Silva, O. F.; Rossi, R. H.; de Maggio, B. Molecular organization, structural orientation, and surface topography of monoacylated $\beta$-cyclodextrins in monolayers at the air-aqueous interface. Langmuir 2008, 24, 7867-7874.

(31) Bauer, M.; Charitat, T.; Fajolles, C.; Fragneto, G.; Daillant, J. Insertion properties of cholesteryl cyclodextrins in phospholipid membranes: a molecular study. Soft Matter 2012, 8, 942-953.

(32) Kurkov, S. V; Loftsson, T. Cyclodextrins. Int. J. Pharm. 2013, 453, 167-180.

(33) Biasutti, M. A.; Abuin, E. B.; Silber, J. J.; Correa, N. M.; Lissi, E. A. Kinetics of reactions catalyzed by enzymes in solutions of surfactants. Adv. Colloid Interface Sci. 2008, 136, 1-24.

(34) Strohmeier, G. A.; Pichler, H.; May, O.; Gruber-Khadjawi, M. Application of designed enzymes in organic synthesis. Chem. Rev. 2011, 111, 4141-4164.

(35) Yang, Z.; Ji, H. 2-Hydroxypropyl- $\beta$-cyclodextrin polymer as a mimetic enzyme for mediated synthesis of benzaldehyde in water. ACS Sustain. Chem. Eng. 2013, 1, 1172-1179.

(36) Dong, Z. Y.; Luo, Q.; Liu, J. Q. Artificial enzymes based on supramolecular scaffolds. Chem. Soc. Rev. 2012, 41, 7890-7908.

(37) Shen, H. M.; Ji, H. B. Biomimetic asymmetric aldol reactions catalyzed by proline derivatives attached to $\beta$-cyclodextrin in water. Tetrahedron Lett. 2012, 53, 3541-3545.

(38) Shen, H. M.; Ji, H. B. Amino alcohol-modified $\beta$-cyclodextrin inducing biomimetic asymmetric oxidation of thioanisole in water. Carbohyd. Res. 2012, 354, 49-58.

(39) Silva, O. F.; Silber, J. J.; de Rossi, R. H.; Correa, N. M.; Fernandez, M. A. On the possibility that cyclodextrins' chiral cavities can be available on AOT n-heptane reverse micelles. A UV-visible and induced circular dichroism study. J. Phys. Chem B 2007, 111, 1070310712.

(40) Harata, K.; Uedaira, H. The circular dichroism spectra of the bcyclodextrin complex with naphthalene derivatives. Bull. Chem. Soc. Jpn. 1975, 48, 375-378.

(41) Adachi, K.; Watarai, H. Site-selective formation of optically active inclusion complexes of alkoxo-subphthalocyanines with $\beta$ cyclodextrin at the toluene/water interface. Chem.-Eur. J. 2006, 12, $4249-4260$

(42) Correa, N. M.; Biasutti, M. A.; Silber, J. J. Micropolarity of reverse micelles of aerosol-OT in n-hexane. J. Colloid Interface Sci. 1995, 172, 71-76.

(43) Silva, O. F.; Fernández, M. A.; Pennie, S. L.; Gil, R. R.; de Rossi, R. H. Synthesis and characterization of an amphiphilic cyclodextrin, a micelle with two recognition sites. Langmuir 2008, 24, 3718-3726.

(44) Salabat, A.; Eastoe, J.; Mutch, K. J.; Rico, F. T. Tuning aggregation of microemulsion droplets and silica nanoparticles using solvent mixtures. J. Colloid Interface Sci. 2008, 318, 244-251.

(45) Sedgwick, M. A.; Trujillo, A. M.; Hendricks, N.; Levinger, N. E.; Crans, D. C. Coexisting aggregates in mixed aerosol OT and cholesterol microemulsions. Langmuir 2011, 27, 948.

(46) Kamlet, M. J.; M. Abboud, J. L.; Abraham, M. H.; Taft, R. W. J. Linear solvation energy relationships. A comprehensive collection of the solvatochromic parameters, $\pi^{*}, \alpha$, and $\beta$, and some methods for simplifying the generalized solvatochromic equation. J. Org. Chem. 1983, 48, 2877-2887.

(47) Jeon, W. S.; Moon, K.; Park, S. H.; Chun, H.; Ko, Y. H.; Lee, J. Y.; Lee, E. S.; Samal, S.; Selvapalam, N.; Rekharsky, M. V.; Sindelar, V.; Sobransingh, D.; Inoue, Y.; Kaifer, A. E.; Kim, K. Complexation of ferrocene derivatives by the cucurbit[7]uril host: a comparative study of the cucurbituril and cyclodextrin host families. J. Am. Chem. Soc. 2005, 127, 12984-12989.

(48) Moyano, F.; Setien, E.; Silber, J. J.; Correa, N. M. Enzymatic hydrolysis of $N$-benzoyl-L-tyrosine $p$-nitroanilide by $\alpha$-chymotrypsin in DMSO-water/AOT $/ n$-heptane reverse micelles. A unique interfacial effect on the enzymatic activity. Langmuir 2013, 29, 8245-8254.

(49) Baruah, B.; Roden, J. M.; Sedgwick, M.; Correa, N. M.; Crans, D. C.; Levinger, N. E. When is water not water? Exploring water confined in large reverse micelles using a highly charged inorganic molecular probe. J. Am. Chem. Soc. 2006, 128, 12758-12765.

(50) Falcone, R. D.; Silber, J. J.; Correa, N. M. What are the factors that control non-aqueous/AOT/ $n$-heptane reverse micelle sizes? A dynamic light scattering study. Phys. Chem. Chem. Phys. 2009, 11, 11096-11100.

(51) Durantini, A. M.; Falcone, R. D.; Silber, J. J.; Correa, N. M. More evidences on the control of the reverse micelles sizes. combination of different techniques as powerful tool to monitor AOT reversed micelles properties. J. Phys. Chem. B 2013, 117, 38183828.

(52) Schönbeck, C.; Westh, P.; Madsen, J. C.; Larsen, K. L.; Städe, L. W.; Holm, R. Hydroxypropyl-substituted $\beta$-cyclodextrins: influence of degree of substitution on the thermodynamics of complexation with tauroconjugated and glycoconjugated bile salts. Langmuir 2010, 26, 17949-17957.

(53) Shimizu, H.; Kaito, A.; Hatano, M. Induced circular dichoroism of $\beta$-cyclodextrin complexes with o-, m-,p- disubstituted benzenes. Bull. Chem. Soc. Jpn. 1981, 54, 513-519.

(54) Shimizu, H.; Kaito, A.; Hatano, M. Induced circular dichroism of $\beta$-cyclodextrin complexes with substituted benzenes. Bull. Chem. Soc. Jpn. 1979, 52, 2678-2684.

(55) Zhang, X.; Nau, W. Chromophore alignment in a chiral host provides a sensitive test for the orientation - intensity rule of induced circular dichroism. Angew. Chem., Int. Ed. 2000, 39, 544-547.

(56) Kodaka, M. A general rule for circular dichroism induced by a chiral. J. Am. Chem. Soc. 1993, 115, 3702-3705.

(57) Krishnan, R.; Rakhi, A. M.; Gopidas, K. R. Study of $\beta$ cyclodextrin-pyromellitic diimide complexation. Conformational analysis of binary and ternary complex structures by induced circular 
dichroism and 2D NMR spectroscopies. J. Phys. Chem. C 2012, 116, 25004-25014.

(58) Bakirci, H.; Zhang, X.; Nau, W. M. Induced circular dichroism and structural assignment of the cyclodextrin inclusion complexes of bicyclic azoalkanes. J. Org. Chem. 2005, 70, 39-46.

(59) Liu, Y.; You, C. C.; Li, B. Synthesis and molecular recognition of novel oligo(ethylenediamino) bridged bis(beta-cyclodextrin)s and their copper(II) complexes: enhanced molecular binding ability and selectivity by multiple recognition. Chem.-Eur. J. 2001, 7, 12811288.

(60) Kuwabara, T.; Ueno, A.; Toda, F. Inclusion complexes and guest-induced color changes of $\mathrm{pH}$-indicator-modified $\beta$-cyclodextrins. J. Phys. Chem. 1994, 6297-6303. 